\title{
Wave propagation retrieval method for metamaterials: Unambiguous restoration of effective parameters
}

\author{
Andryieuski, Andrei; Malureanu, Radu; Lavrinenko, Andrei
}

Published in:

Physical Review B Condensed Matter

Link to article, DOI:

10.1103/PhysRevB.80.193101

Publication date:

2009

Document Version

Publisher's PDF, also known as Version of record

Link back to DTU Orbit

Citation (APA):

Andryieuski, A., Malureanu, R., \& Lavrinenko, A. (2009). Wave propagation retrieval method for metamaterials: Unambiguous restoration of effective parameters. Physical Review B Condensed Matter, 80(19), 193101. https://doi.org/10.1103/PhysRevB.80.193101

\section{General rights}

Copyright and moral rights for the publications made accessible in the public portal are retained by the authors and/or other copyright owners and it is a condition of accessing publications that users recognise and abide by the legal requirements associated with these rights.

- Users may download and print one copy of any publication from the public portal for the purpose of private study or research.

- You may not further distribute the material or use it for any profit-making activity or commercial gain

- You may freely distribute the URL identifying the publication in the public portal 


\title{
Wave propagation retrieval method for metamaterials: Unambiguous restoration of effective parameters
}

\begin{abstract}
Andrei Andryieuski, * Radu Malureanu, and Andrei V. Lavrinenko
DTU Fotonik, Technical University of Denmark, Oersteds plads 343, Kongens Lyngby, DK-2800, Denmark

(Received 10 September 2009; revised manuscript received 12 October 2009; published 3 November 2009)

In this Brief report we propose a direct method of effective-parameters restoration that is based on the wave propagation phenomenon. It is easy in implementation, has no unambiguity in retrieving effective properties and is applicable to thick metamaterial (MTM) slabs. The method is validated on the case studies of fishnet, split cube in carcass, and Jerusalem cross MTMs. The constraints of the method are designated.
\end{abstract}

DOI: 10.1103/PhysRevB.80.193101

PACS number(s): 78.20.Ci, 41.20.Jb, 42.25.Bs

Metamaterials are a new emerging field in photonics due to extravagant properties that can be obtained with engineering of their structure. After some successful proof-ofprinciple experiments with the negative-index behavior, MTMs are moving now toward new frontiers, for example, having material constants either close to zero or very large, exhibiting isotropy or extreme anisotropy, dumping or enhancing spatial dispersion. It provides great opportunities to control the light propagation. The potential applications of MTMs span from invisibility cloaks ${ }^{1}$ to super resolution lenses $^{2}$ and nanocouplers ${ }^{3}$ but are not limited to them.

Assigning certain values to some parameters is the mean by which the properties of the MTM can be characterized in the universal mode. In the cases when effective parameters (refractive index $n$, impedance $Z$, permittivity $\varepsilon$, and permeability $\mu$ ) can be introduced in the correct way, ${ }^{4}$ a reliable retrieval procedure is on demand. The existing methods can be divided into following groups: (1) reflection/transmission ( $S$ parameters) based. ${ }^{5}$ The procedure is easy to implement and widely used so we refer to it as the standard method (SM). Initially proposed for normal incidence on the isotropic metamaterial ${ }^{5,6}$ the method was modified for inclined incidence, ${ }^{7}$ bianisotropic, ${ }^{8,9}$ and chiral metamaterials. ${ }^{10}$ (2) Field averaging. ${ }^{11,12}$ In this case the fields $(E, D, H, B)$ in the unit cell are averaged on some surfaces and lines and the effective properties are found according to constitutive relations $D=\varepsilon E$ and $B=\mu H$. (3) Quasimode theory. ${ }^{13}$ This method is based on the maximization of optical density of states for a MTM while changing $\varepsilon$ and $\mu$ of the surrounding medium. (4) Wave propagation phenomena. ${ }^{14}$ It was proposed to match the simulated fields inside and outside of a thin MTM slab with the fields theoretically predicted by formulas. Fitting parameters in this case are refractive index and impedance.

All the methods have case-specific limitations, e.g., SM suffers from the notorious "branch" problem and works well only for thin slabs that is not enough in general describing a bulk MTM. ${ }^{15}$ Other methods have shorthands in complexity of realization or extensive numerical load.

The method we propose in this Brief Report is based on the wave propagation and we refer to it as the wave propagation retrieval method (WPRM). In some sense it exploits and elaborates ideas revealed in Ref. 14 but WPRM has significant differences. We propose to monitor wave amplitude and phase during its propagation through a thick MTM slab so the wave reflected back from the rear interface is negligible and formation of any standing wave is avoided. The retrieval formulas for $n$ become easy to use and unambiguous. The effective parameters derived from the thick-layer simulation are automatically bulk. Impedance is retrieved from the reflection problem at the input interface.

The simulation geometry assumes the MTM slab infinite in $x$ and $y$ directions and semi-infinite in $z$ direction of propagation (Fig. 1). The normal incidence of the wave occurs from vacuum. The fields inside each unit cell have complicated spatial distribution so in order to apply the effectivemedium description we should abstract from composite intrinsic structure and regard it as a bulk homogeneous material. Thus we average the components of complex field vectors in each of the unit cells. We chose the electric field but the procedure can be easily reformulated for the magnetic field. One more assumption we utilize here for the sake of simplicity is the scalar procedure of restoration, which means linear polarization of the fields with designs exhibiting no chirality or anisotropy in the direction of propagation.

We describe the light wave of frequency $\omega$ as a modulated plane wave

$$
E(x, y, z)=E_{0} u(x, y, z) \exp \left(-i k^{\prime} z-k^{\prime \prime} z\right),
$$

where $u(x, y, z)$ is a modulation function periodic in $z$ direction with the same period $a$ as MTM, $k^{\prime}=n^{\prime} \omega / c$ and $k^{\prime \prime}$ $=n^{\prime \prime} \omega / c$ are the real and imaginary parts of the wave number connected with the real $n^{\prime}$ and imaginary $n^{\prime \prime}$ parts of the refractive index. Function $u(x, y, z)$ changes quickly over the unit cell comparing with the exponential ansatz in Eq. (1). Assuming the slow variation in the phase, which is valid under the generally accepted homogeneity condition $\lambda_{\text {eff }}$

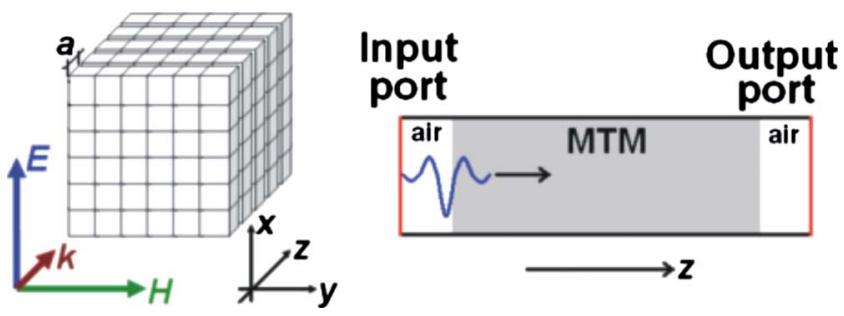

FIG. 1. (Color online) Wave propagation simulation configuration. 


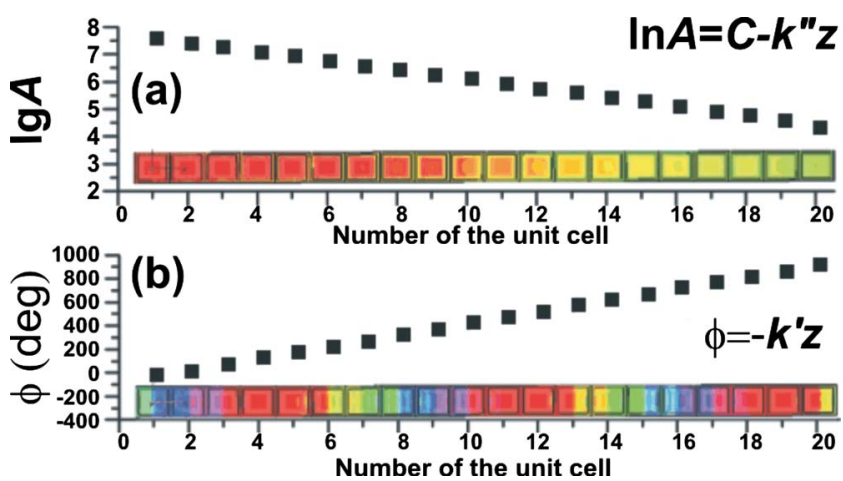

FIG. 2. (Color online) An example of (a) amplitude and (b) phase of the averaged field distribution over 20 unit cells of the split cube in carcass design for the negative refractive-index regime. Color scales used in both cases for demonstration of the averaged amplitudes and phases are not equivalent.

$>4 a$, the averaged over the $N$ th unit-cell electric field is equal to

$$
\langle E\rangle_{N} \approx E_{0} \exp \left(-k^{\prime \prime} z_{N}\right) \exp \left(-i k^{\prime} z_{N}\right)=A \exp (-i \phi),
$$

where $z_{N}=(N-1 / 2) a$ is the coordinate of the $N$ th unit-cell center, $A=\left|\langle E\rangle_{N}\right|$ and $\phi=\arg \left(\langle E\rangle_{N}\right)$ are the amplitude and phase of the field averaged over the $N$ th unit-cell volume.

Both parts of the refractive index are straightforwardly derived assuming linear changes in phase $\Delta \phi$ and amplitude $\operatorname{logarithm} \Delta \ln A$ with a number of unit cells $\Delta N$ (Fig. 2)

$$
n^{\prime}=-\frac{\Delta \phi}{\Delta N} \frac{c}{a \omega}, \quad n^{\prime \prime}=-\frac{\Delta \ln A}{\Delta N} \frac{c}{a \omega} .
$$

Imposing semi-infinity in $z$ direction implies only one vacuum-MTM interface. In this case impedance $Z$ can be restored in the unique way from the reflection $r$ at the single interface

$$
Z=\frac{1+r}{1-r} .
$$

In numerical realization of the method we use a thick but finite MTM. So, the impedance formula (4) can be justified by the presence of absorption. Knowing the refractive index and impedance one can easily obtain permittivity and permeability $\varepsilon=n / Z, \mu=n Z$.

We performed the time-domain simulations of the wave propagation inside thick MTM slabs (typically 20-50 periods) using CST MICROWAVE STUDIO 2009. For broadband excitation a short modulated Gaussian pulse was chosen. As a reference we used the effective parameters retrieved with SM. Reflection/transmission through three MTM layers were simulated with the CST frequency-domain solver. We found out that results retrieved with the WPRM are equal to that retrieved with the SM in the full pulse bandwidth (see below). An illustration of the WPRM realization for a split cube in carcass (SCiC) structure is shown in Fig. 2.

WPRM has several clear advantages. First, it does not experience the branch problem. Second, WPRM does not suffer from such simulation problems as low transmission distorted with noise and long simulation time for sufficient

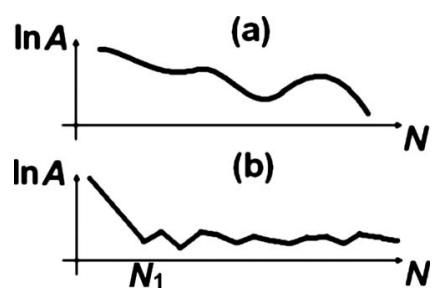

FIG. 3. Possible problematic cases of simulated average amplitude distribution inside the MTM slab: (a) standing-wave formation due to the back interface reflection and slow wave attenuation; (b) large wave attenuation and numerical noise.

spectra resolution as it uses the amplitude and phase distribution inside the structure. Third, the method works well for thick slabs thus giving bulk effective parameters values. Finally, to avoid the branch problem restoring the effective parameters with SM one should always use the continuity of the spectra and therefore broadband simulation. There is no difference for WPRM whether to use broadband or narrowband spectra.

WPRM also has some constraints. We depict two possible variants of amplitude behavior (Fig. 3). With very low absorption (or with a thin slab) reflection from the back interface leads to standing-wave formation [Fig. 3(a)]. To retrieve $n$ and $Z$ correctly we should make the MTM slab thicker and take into account only the first linear part of the graph. Another option is to trace the impulse dynamics and terminate calculations as soon as back-reflected pulse begins to form out.

In the other limit of too strong attenuation the signal amplitude is quickly reduced to the noise level [Fig. 3(b)]. Noise data should be excluded for restoration by taking only first $N_{1}$ cells.

To quantify the limitations of WPRM we simulated "the worst case:" a homogenous dielectric slab of thickness $d$ with predefined refractive index $n$ and impedance $Z=1$ with a perfect mirror (perfect electric conductor boundary condition) on the other side of the slab. Numerical simulations were performed until the total electromagnetic energy stored in the system dropped down to $-80 \mathrm{~dB}$ with respect to the maximum level. It is obvious that not the value of the refractive index itself but rather terms in exponentials in Eq. (2) govern phase and amplitude changes. We calculated the relative error of restored $n$ for different $n^{\prime} k_{0} d$ and $n^{\prime \prime} k_{0} d$, where $k_{0}=\omega / c$ is the wave number in vacuum. In the case of fast amplitude decay [Fig. 3(b)] we should consider $d$ as the distance at which $\ln A$ reaches the noise level. We identified the optimal region where the total relative error is less than $1 \%$. The conclusion is that in the worst case WPRM is applicable with a relative error less than $1 \%$ for $-45 \leq n^{\prime} k_{0} d \leq 45$ and $5 \leq n^{\prime \prime} k_{0} d \leq 56$. In the absence of the perfect reflector at the second interface, like as in conventional MTM cases, these limits are extended much further.

The range of applicability of WPRM can be extended to the case of low-loss structures if we excite the MTM slab with a short Gaussian pulse and stop the simulation when the center of the pulse reaches the rear interface. So the reflected wave exists only near the rear interface. This part of data must be omitted while retrieving refractive index. As a short- 

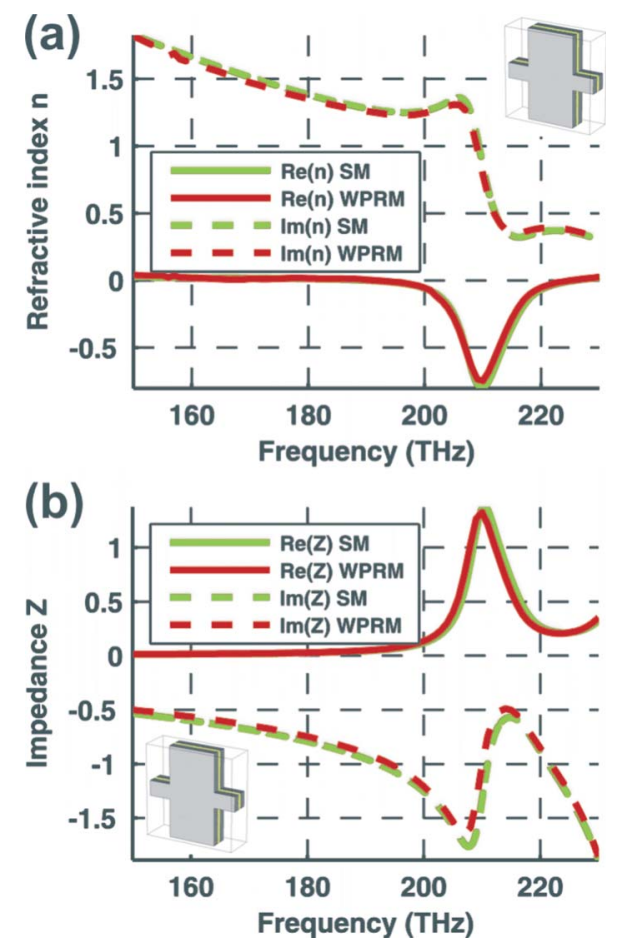

FIG. 4. (Color online) Refractive index (a) and impedance (b) of the fishnet structure restored with WPRM [red (black)] and SM [green (light gray)]. Real and imaginary parts are marked with solid and dashed lines, respectively. This color and line convention will be respected in all subsequent figures.

coming we should note that such simulation is possible only for MTMs with low dispersion and requires additional estimation of the time the pulse travels through the slab.

To make a comprehensive validation of the WPRM we chose MTM designs operating at different frequencies, i.e., in optical and microwave ranges. The SM (Ref. 5) results were used for reference.

The fishnet structure ${ }^{16}$ is a classical example of the optical negative index MTM with high transmittivity. For the WPRM test we took design parameters from Ref. 15. Effective parameters $n$ and $Z$ restored with both WPRM and SM are in a perfect agreement (Fig. 4). This example also shows that WPRM works well for both single negative and double negative metamaterials as fishnet is single negative below $190 \mathrm{THz}$.

The SCiC structure is generic to the split-cube-in-cage design. ${ }^{17}$ Its remarkable property is the fast convergence (after only one layer) of the effective parameters to bulk values. ${ }^{17}$ The structure possesses cubic symmetry that guarantees its polarization insensitivity. As a drawback, SCiC has high absorption and thus quite low transmittivity. The SCiC unit cell consists of two silver parts embedded in silica $(n$ $=1.5$ ). Silver is regarded as a Drude metal with plasma and collision frequency as in Ref. 16. Both WPRM and SM provide very similar results (Fig. 5). Differences in the retrieved impedance on frequencies above 190THz [Fig. 5(b)] come out from intensive dipole resonances of split cubes and failure of the effective homogeneity approach. ${ }^{17}$

Recently Wang et al. ${ }^{18}$ has reported a three-dimensional MTM design for the microwave region claiming isotropic
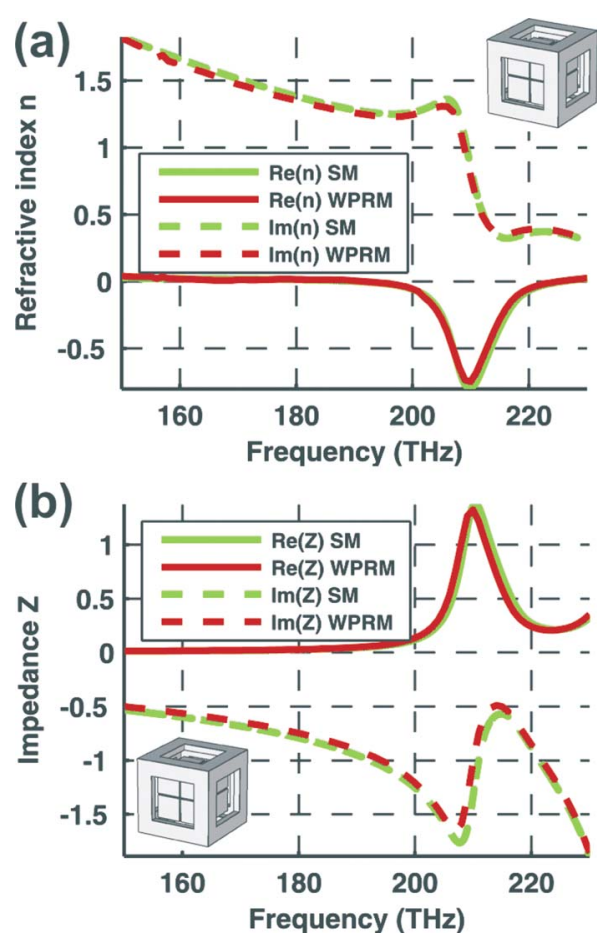

FIG. 5. (Color online) Split cube in carcass effective parameters: (a) refractive index and (b) impedance.

broadband left handedness. Design consists of Jerusalem crosses arranged in a cubic lattice (see inset in Fig. 6). As the reported losses in this MTM are relatively low it was a nice opportunity to check WPRM. All the design parameters were taken the same as in Ref. 18. To our surprise WPRM shows no any negative refractive index in the specified region
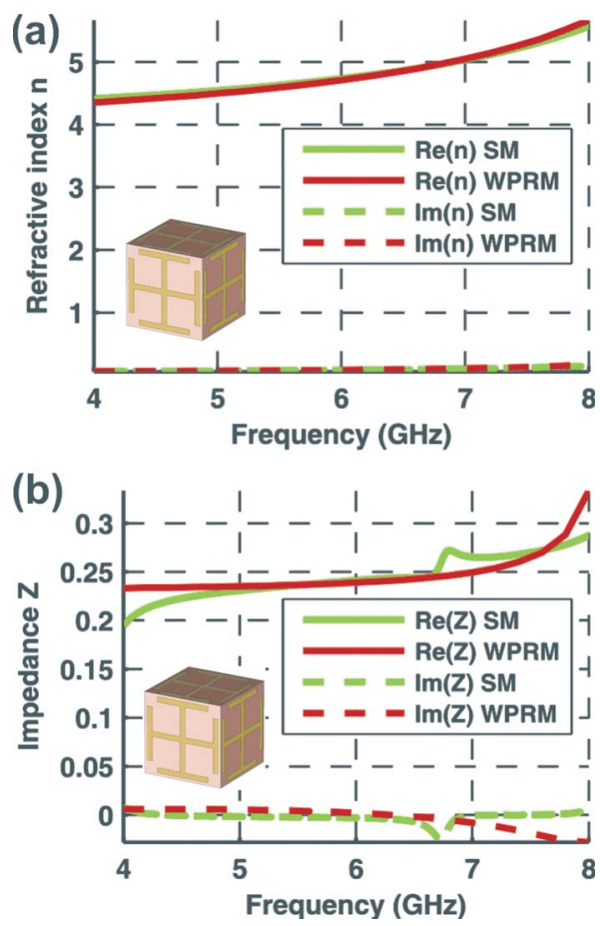

FIG. 6. (Color online) Jerusalem crosses MTM effective parameters: (a) refractive index and (b) impedance. 
(around 6GHz) [Fig. 6(a)]. WPRM results perfectly coincide with the SM results if one chooses the correct branch. Feasibility of no negative index behavior is easy to explain if we take into account that real and imaginary parts of $n$ must obey the Kramers-Kronig relations. ${ }^{19}$ Small and smooth imaginary part corresponds to smooth changes in the real part in contrary to sudden abrupt jumps presented. ${ }^{18}$ This is an important message concerning WPRM functionality to avoid errors and misunderstanding in the refractive index restoration and interpretation.

A kink in the impedance spectrum at $6.8 \mathrm{GHz}$ restored with SM [Fig. 6(b)] appears due to the finiteness of the system. Additional studies showed that it changes position with the number of layers (thickness) always being exactly at the frequency of the corresponding Fabry-Perot resonance. No such problem is connected with WPRM as multiple reflections from the slab interfaces are successfully avoided.

Low value of $\operatorname{Im}(n)$ in Jerusalem crosses MTM is restored correctly [Fig. 6(a)] that confirms applicability of WPRM not only to lossy structures but also to low-loss ones. To sum up, we propose the effective parameters retrieval method based on the wave propagation phenomenon and validate its applicability to lossy and low-loss metamaterials on the case studies in microwaves and optical ranges. The main advantages of WPRM are: unambiguous retrieving of the effective parameters, easy straightforward implementation, applicability to thick (bulky) MTM slabs, and to broad variety of designs, absence of spurious effects connected with the Fabry-Perot resonances. WPRM can be applied whenever the effectivemedium approximation is valid. In principle, WPRM can be extended to anisotropic media or media with a nonlocal response as soon as required observable features of the wave can be detected numerically.

The authors acknowledge partial support from the Danish Research Council for Technology and Production Sciences via the NIMbus project and COST Action MP0702. *andra@fotonik.dtu.dk

${ }^{1}$ D. Schurig, J. J. Mock, B. J. Justice, S. A. Cummer, J. B. Pendry, A. F. Starr, and D. R. Smith, Science 314, 977 (2006).

${ }^{2}$ J. B. Pendry, Phys. Rev. Lett. 85, 3966 (2000).

${ }^{3}$ A. Degiron, D. R. Smith, J. J. Mock, B. J. Justice, and J. Gollub, Appl. Phys. A: Mater. Sci. Process. 87, 321 (2007).

${ }^{4}$ C. R. Simovski and S. A. Tretyakov, Phys. Rev. B 75, 195111 (2007).

${ }^{5}$ D. R. Smith, S. Schultz, P. Markos, and C. M. Soukoulis, Phys. Rev. B 65, 195104 (2002).

${ }^{6}$ X. Chen, T. M. Grzegorczyk, B. I. Wu, J. Pacheco, and J. A. Kong, Phys. Rev. E 70, 016608 (2004).

${ }^{7}$ C. Menzel, C. Rockstuhl, T. Paul, F. Lederer, and T. Pertsch, Phys. Rev. B 77, 195328 (2008).

${ }^{8}$ X. Chen, B.-I. Wu, J. A. Kong, and T. M. Grzegorczyk, Phys. Rev. E 71, 046610 (2005).

${ }^{9}$ Z. Li, K. Aydin, and E. Ozbay, Phys. Rev. E 79, 026610 (2009).

${ }^{10}$ C. Menzel, C. Rockstuhl, T. Paul, and F. Lederer, Appl. Phys. Lett. 93, 233106 (2008).
${ }^{11}$ D. R. Smith and J. B. Pendry, J. Opt. Soc. Am. B 23, 391 (2006).

${ }^{12}$ J.-M. Lerat, N. Mallejac, and O. Acher, J. Appl. Phys. 100, 084908 (2006).

${ }^{13}$ S. Sun, S. T. Chui, and L. Zhou, Phys. Rev. E 79, 066604 (2009).

${ }^{14}$ B. I. Popa and S. A. Cummer, Phys. Rev. B 72, 165102 (2005).

${ }^{15}$ C. Rockstuhl, T. Paul, F. Lederer, T. Pertsch, T. Zentgraf, T. P. Meyrath, and H. Giessen, Phys. Rev. B 77, 035126 (2008).

${ }^{16}$ G. Dolling, C. Enkrich, and M. Wegener, Opt. Lett. 31, 1800 (2006).

${ }^{17}$ A. Andryieuski, C. Menzel, C. Rockstuhl, R. Malureanu, and A. V. Lavrinenko, J. Opt. A, Pure Appl. Opt. 11, 114010 (2009).

${ }^{18}$ J. F. Wang, S. B. Qu, Z. Xu, Z. T. Fu, H. Ma, and Y. M. Yang, J. Phys. D 42, 155413 (2009).

${ }^{19}$ V. Lucarini, J. Saarinen, K.-E. Peiponen, and E. Vartiainen, Kramers-Kronig Relations in Optical Materials Research (Springer-Verlag, Berlin, 2005). 